\title{
Spatial Central Configurations with Two Twisted Regular 4-Gons
}

\author{
Sen Zhang ${ }^{1,2}$ and Furong Zhao ${ }^{3}$ \\ ${ }^{1}$ School of Electronic Engineering, UEST Of China, Chengdu 610054, China \\ ${ }^{2}$ Department of Mathematics, Yibin University, Sichuan, 644007, China \\ ${ }^{3}$ Department of Mathematics and Computer Science, Mianyang Normal University, Mianyang, Sichuan 621000, China
}

Correspondence should be addressed to Furong Zhao; zhaofurong2006@163.com

Received 23 May 2013; Accepted 19 September 2013

Academic Editor: Orazio Descalzi

Copyright (C) 2013 S. Zhang and F. Zhao. This is an open access article distributed under the Creative Commons Attribution License, which permits unrestricted use, distribution, and reproduction in any medium, provided the original work is properly cited.

\begin{abstract}
We study the configuration formed by two squares in two parallel layers separated by a distance. We picture the two layers horizontally with the $z$-axis passing through the centers of the two squares. The masses located on the vertices of each square are equal, but we do not assume that the masses of the top square are equal to the masses of the bottom square. We prove that the above configuration of two squares forms a central configuration if and only if the twist angle is equal to $k \pi / 2$ or $(\pi / 4+k \pi / 2)$ $(k=1,2,3,4)$.
\end{abstract}

\section{Introduction and Main Results}

This paper uses the same notations as [1]. The Newtonian $N$ body problems $[2,3]$ concern the motions of $N$ particles with masses $m_{j} \in R^{+}$and positions $q_{j} \in R^{3}(j=1,2, \ldots, N)$. The motion is governed by Newton's second law and the Universal law:

$$
m_{j} \ddot{q}_{j}=\frac{\partial U(q)}{\partial q_{j}}
$$

where $q=\left(q_{1}, q_{2}, \ldots, q_{N}\right)$ with the Newtonian potential:

$$
U(q)=\sum_{1 \leqslant j<k \leqslant N} \frac{m_{j} m_{k}}{\left|q_{j}-q_{k}\right|} .
$$

Consider the space

$$
X=\left\{q=\left(q_{1}, q_{2}, \ldots, q_{N}\right) \in R^{3 N}: \sum_{j=1}^{N} m_{j} q_{j}=0\right\} ;
$$

that is, suppose that the center of mass is fixed at the origin of the origin of the coordinate axis, because the potential is singular when two particles have the same position. It is natural to assume that the configuration avoids the collision set $\Delta=\left\{q: q_{j}=q_{k}\right.$ for some $\left.k \neq j\right\}$. The set $X \backslash \Delta$ is called the configuration space.

Definition 1 (see $[2,3])$. A configuration $q=\left(q_{1}, q_{2}, \ldots\right.$, $\left.q_{N}\right) \in X \backslash \Delta$ is called a central configuration if there exists a constant $\lambda$ such that

$$
\sum_{j=1, j \neq k}^{N} \frac{m_{j} m_{k}}{\left|q_{j}-q_{k}\right|^{3}}\left(q_{j}-q_{k}\right)=-\lambda m_{k} q_{k}, \quad 1 \leqslant k \leqslant N .
$$

The value of constant $\lambda$ in (4) is uniquely determined by

$$
\lambda=\frac{U}{I},
$$

where

$$
I=\sum_{k=1}^{N} m_{k}\left|q_{k}\right|^{2} .
$$

Consider the configuration in $R^{3}$ consisting of two layer regular $N$-gons $(N \geqslant 2)$ with distance $h \geqslant 0$. It is assumed that the lower layer regular $\mathrm{N}$-gons lie in horizontal plane, and the upper regular $N$-gons parallel the lower 
one, and $z$-axis passes through both centers of two regular $N$-gons. Suppose that the lower layer particles have masses $m_{1}, m_{2}, \ldots, m_{N}$ and the upper layer particles have masses $\widetilde{m}_{1}, \widetilde{m}_{2}, \ldots, \widetilde{m}_{N}$, respectively; then these assumptions can be interpreted more precisely by the following. Let $\rho_{k}$ denote for all $N$ complex roots of unity, that is,

$$
\rho_{k}=\exp \left(\frac{2 \pi k}{N} i\right) \text {. }
$$

Let

$$
\tilde{\rho}_{k}=a \rho_{k} e^{i \varphi}
$$

where $a>0, i=\sqrt{-1}, 0 \leqslant \varphi \leqslant 2 \pi$, and $\varphi$ is called twist angle. It is assumed that $m_{k}(1 \leqslant k \leqslant N)$ locates at the vertex $q_{k}$ of the lower layer regular $N$-gons; $\widetilde{m}_{k}(1 \leqslant k \leqslant N)$ locates on the vertex $\bar{q}_{k}$ of the upper layer regular $N$-gons:

$$
\begin{aligned}
& q_{k}=\left(\rho_{k}, 0\right), \\
& \widetilde{q}_{k}=\left(\widetilde{\rho}_{k}, h\right),
\end{aligned}
$$

where $h \geq 0$ is the distance between the two layers. Then the center of masses is

$$
z_{0}=\sum_{j=1}^{N} \frac{\left(m_{j} q_{j}+\widetilde{m}_{j} \widetilde{q}_{j}\right)}{M},
$$

where

$$
M=\sum_{j=1}^{N}\left(m_{j}+\widetilde{m}_{j}\right) .
$$

Let

$$
\begin{aligned}
& P_{K}=q_{k}-z_{0}, \\
& \widetilde{P}_{K}=\tilde{q}_{k}-z_{0} .
\end{aligned}
$$

If $m_{1}, m_{2}, \ldots, m_{N}$ and $\widetilde{m}_{1}, \widetilde{m}_{2}, \ldots, \widetilde{m}_{N}$ form a central configuration, then there is $\lambda \in R^{+}$such that

$$
\begin{aligned}
& \sum_{j \neq k}^{N} m_{j} \frac{P_{j}-P_{k}}{\left|P_{j}-P_{k}\right|^{3}}+\sum_{j=1}^{N} \widetilde{m}_{j} \frac{\widetilde{P}_{j}-P_{k}}{\left|\widetilde{P}_{j}-P_{k}\right|^{3}}=-\lambda P_{k}, \quad 1 \leqslant k \leqslant N, \\
& \sum_{j=1}^{N} m_{j} \frac{P_{j}-\widetilde{P}_{k}}{\left|P_{j}-\widetilde{P}_{k}\right|^{3}}+\sum_{j \neq k}^{N} \widetilde{m}_{j} \frac{\widetilde{P}_{j}-\widetilde{P}_{k}}{\left|\widetilde{P}_{j}-\widetilde{P}_{k}\right|^{3}}=-\lambda \widetilde{P}_{k}, \quad 1 \leqslant k \leqslant N .
\end{aligned}
$$

Under the case that twist angle $\varphi=0$, Moeckel-Simo proved.

Theorem 2 (see [4]). If $N<473$, there is a unique pair of spatial central configurations of parallel regular $N$-gons. If $N \geq 473$, there is no such central configuration for $b<b_{0}(N)$, where $b$ is the mass ratio. At a unique pair bifurcates from the planar central configuration with the smaller masses on the inner polygon. This remains the unique pair of spatial central configurations until $b=1 / b_{0}$, where a similar bifurcation occurs in reverse, so that $b>1 / b_{0}$, and only the planar central configurations remain.
Xie et al. [5] studied the necessary conditions for the masses of two layer regular polygon central configurations in $R^{3}$, and they proved the following theorems.

Theorem 3. Under the assumptions of (9), if $m_{1}, m_{2}, \ldots, m_{N}$ and $\widetilde{m}_{1}, \widetilde{m}_{2}, \ldots, \widetilde{m}_{N}$ form a central configuration, then

(i)

$$
\lambda=\frac{M}{N} \sum_{j=1}^{N} \frac{1}{\left|q_{j}-\tilde{q}_{N}\right|^{3}},
$$

(ii) for $N \geqslant 2(\varphi \neq \pi / 2$, when $N=2)$,

$$
m_{1}=m_{2}=\cdots=m_{N}, \quad \widetilde{m}_{1}=\widetilde{m}_{2}=\cdots=\widetilde{m}_{N} .
$$

Without loss of generality, suppose that $\widetilde{m}_{j}=b m_{j}$ and $N \geqslant 2$ $(\varphi \neq \pi / 2$, when $N=2)$, under the above assumptions, there are four parameters, and ratio $b$ of masses ratio, a of radius of two regular $N$-gons, the distance $h$ between two layers, and the phase difference $\varphi$. As for these parameters, Xie et al. [5] proved.

Theorem 4. Under the assumptions of (9) and $\widetilde{m}_{j}=b m_{j}$, then $m_{1}, m_{2}, \ldots, m_{N}$ and $\widetilde{m}_{1}, \widetilde{m}_{2}, \ldots, \widetilde{m}_{N}$ form a central configuration if and only if the parameters $b, a, h$, and $\varphi$ satisfy the following relationships:

$$
\begin{aligned}
& \frac{\lambda}{M}=\frac{1}{N(1+b)}\left(\sum_{j \neq N} \frac{1-\rho_{j}}{\left|q_{j}-q_{N}\right|^{3}}+\sum_{j} \frac{b\left(1-a \rho_{j} e^{i \varphi}\right)}{\left|\tilde{q}_{j}-q_{N}\right|^{3}}\right), \\
& \frac{\lambda}{M}=\frac{1}{N} \sum_{j=1}^{N} \frac{1}{\left|q_{j}-\widetilde{q}_{N}\right|^{3}}, \\
& \frac{\lambda}{M}=\frac{e^{-i \varphi}}{N a(1+b)}\left(\sum_{j} \frac{a e^{i \varphi}-\rho_{j}}{\left|q_{j}-\tilde{q}_{N}\right|^{3}}+\sum_{j \neq N} \frac{b\left(a-a \rho_{j} e^{i \varphi}\right)}{\left|\tilde{q}_{j}-\tilde{q}_{N}\right|^{3}}\right) .
\end{aligned}
$$

Zhang and Zhu [1] proved the sufficient conditions for special cases $a=1, b=1$, and $\varphi=\pi / N$.

Theorem 5. Under the assumptions of (9) and (15) and $\widetilde{m}_{j}=$ bm $_{j}$ if $a=1, b=1$, and $\varphi=\pi / N$, then for every $N$ there exists a unique central configuration.

When $h=0$, Bang and Elmabsout [6] study the twist angle.

Theorem 6. Let $\Pi_{1}$ (resp., $\Pi_{2}$ ) be a regular $N$-gon centred around a mass $m_{0}$ at $O, m_{1}$ being at each of its vertices (resp., $m_{2}$ ). Then $m_{0}, \Pi_{1}$, and $\Pi_{2}$ are relative equilibriums if and only if they are homothetic or cursed with an angle equal to $(\pi / N)$ (and suitable ratio of radii).

A natural interesting problem is that whether (9) form a central configuration for $h>0$ if and only if twist angle $\varphi=0$ or $\pi / N$. In this paper, one will prove the necessary condition of twisted angle $\varphi$ for a special case $N=4$.

Theorem 7. Under the assumptions of (9) and (15) and $\widetilde{m}_{j}=$ bm $_{j}$ if $N=4$, then $\varphi=\pi / 4+(\pi / 2) k$ or $\varphi=(\pi / 2) k,(k=$ $0,1,2,3)$. 


\section{The Proof of Theorem 7}

2.1. Some Lemmas. We need three lemmas.

Lemma 1 (see [7]). For $N \geq 3$ and $\widetilde{m}_{1}=\widetilde{m}_{2}=\cdots=\widetilde{m}_{N}$, if $\widetilde{m}_{1}, \widetilde{m}_{2}, \ldots, \widetilde{m}_{N}$ locate on vertices of a regular polygon, then they form a central configuration.

One has $m_{1}^{*}=m_{2}^{*}=m_{3}^{*}=m_{4}^{*}$. Also $m_{1}^{*}, m_{2}^{*}, m_{3}^{*}$, and $m_{4}^{*}$ are located on vertices of a square $S$; $q_{i}^{*}$ is the position $m_{i}^{*} \quad(i=$ $1,2,3,4)$ and $q^{*}$ is the position of $m^{*}$. Plane $P$ contains the square $S$. One has $F_{*}=\sum_{i=1}^{4} m_{i}^{*} m^{*}\left(q_{i}^{*}-q^{*}\right) /\left|q_{i}^{*}-q^{*}\right|^{3}$.

Lemma 2. $F_{*}$ 's projection on $P$ is directed toward the center of the square if and only if the point $m^{*}$ is on a vertical plane of symmetry.

2.2. The Proof of Lemma 2. Without loss of generality, suppose that $m_{1}^{*}=m_{2}^{*}=m_{3}^{*}=m_{4}^{*}=1, q_{1}^{*}=(1,0,0), q_{2}^{*}=$ $(0,1,0), q_{3}^{*}=(-1,0,0), q_{4}^{*}=(0,-1,0)$, and $q^{*}=(\rho \cos \theta$, $\rho \sin \theta, h)$, where $\rho>0$. Consider the following:

$$
\begin{aligned}
F_{*}= & \frac{m^{*}(1-\rho \cos \theta,-\rho \sin \theta,-h)}{\left|1+\rho^{2}+h^{2}-2 \rho \cos \theta\right|^{3 / 2}} \\
& +\frac{m^{*}(-\rho \cos \theta, 1-\rho \sin \theta,-h)}{\left|1+\rho^{2}+h^{2}-2 \rho \sin \theta\right|^{3 / 2}} \\
& +\frac{m^{*}(-1-\rho \cos \theta,-\rho \sin \theta,-h)}{\left|1+\rho^{2}+h^{2}+2 \rho \cos \theta\right|^{3 / 2}} \\
& +\frac{m^{*}(-\rho \cos \theta,-1-\rho \sin \theta,-h)}{\left|1+\rho^{2}+h^{2}+2 \rho \sin \theta\right|^{3 / 2}} .
\end{aligned}
$$

If $F_{*}$ 's projection on $P$ is directed toward the center of the square, there exists $\lambda^{*}>0$ such that

$$
\begin{aligned}
& \frac{m^{*}(1-\rho \cos \theta)}{\left|1+\rho^{2}+h^{2}-2 \rho \cos \theta\right|^{3 / 2}}+\frac{m^{*}(-\rho \cos \theta)}{\left|1+\rho^{2}+h^{2}-2 \rho \sin \theta\right|^{3 / 2}} \\
& \quad+\frac{m^{*}(-1-\rho \cos \theta)}{\left|1+\rho^{2}+h^{2}+2 \rho \cos \theta\right|^{3 / 2}} \\
& \quad+\frac{m^{*}(-\rho \cos \theta)}{\left|1+\rho^{2}+h^{2}+2 \rho \sin \theta\right|^{3 / 2}} \\
& =-\lambda^{*} \rho \cos \theta,
\end{aligned}
$$$$
=\frac{m^{*}(-\rho \sin \theta)}{\left|1+\rho^{2}+h^{2}-2 \rho \cos \theta\right|^{3 / 2}}
$$$$
+\frac{m^{*}(1-\rho \sin \theta)}{\left|1+\rho^{2}+h^{2}-2 \rho \sin \theta\right|^{3 / 2}}
$$$$
+\frac{m^{*}(-\rho \sin \theta)}{\left|1+\rho^{2}+h^{2}+2 \rho \cos \theta\right|^{3 / 2}}
$$$$
+\frac{m^{*}(-1-\rho \sin \theta)}{\left|1+\rho^{2}+h^{2}+2 \rho \sin \theta\right|^{3 / 2}}
$$$$
=-\lambda^{*} \rho \sin \theta \text {. }
$$

If $\theta=(\pi / 2) k, m^{*}$ is on a vertical plane of symmetry.

When $\theta \neq(\pi / 2) k$, multiplying both sides of (18) and (19), respectively, by $\sin \theta$ and $\cos \theta$, we have

$$
\begin{aligned}
& \frac{\sin \theta-\rho \cos \theta \sin \theta}{\left|1+\rho^{2}+h^{2}-2 \rho \cos \theta\right|^{3 / 2}}+\frac{-\rho \cos \theta \sin \theta}{\left|1+\rho^{2}+h^{2}-2 \rho \sin \theta\right|^{3 / 2}} \\
& \quad+\frac{-\sin \theta-\rho \cos \theta \sin \theta}{\left|1+\rho^{2}+h^{2}+2 \rho \cos \theta\right|^{3 / 2}} \\
& \quad+\frac{-\rho \cos \theta \sin \theta}{\left|1+\rho^{2}+h^{2}+2 \rho \sin \theta\right|^{3 / 2}} \\
& =-\lambda^{*} \rho \cos \theta \sin \theta, \\
& \frac{-\rho \cos \theta \sin \theta}{\left|1+\rho^{2}+h^{2}-2 \rho \cos \theta\right|^{3 / 2}+\frac{\cos \theta-\rho \cos \theta \sin \theta}{\left|1+\rho^{2}+h^{2}-2 \rho \sin \theta\right|^{3 / 2}}} \\
& +\frac{-\rho \cos \theta \sin \theta}{\left|1+\rho^{2}+h^{2}+2 \rho \cos \theta\right|^{3 / 2}} \\
& +\frac{-\cos \theta-\rho \cos \theta \sin \theta}{\left|1+\rho^{2}+h^{2}+2 \rho \sin \theta\right|^{3 / 2}} \\
& =-\lambda^{*} \rho \cos \theta \sin \theta .
\end{aligned}
$$

By (20) we have

$$
\begin{aligned}
& \frac{\sin \theta}{\left|1+\rho^{2}+h^{2}-2 \rho \cos \theta\right|^{3 / 2}}+\frac{-\cos \theta}{\left|1+\rho^{2}+h^{2}-2 \rho \sin \theta\right|^{3 / 2}} \\
& +\frac{-\sin \theta}{\left|1+\rho^{2}+h^{2}+2 \rho \cos \theta\right|^{3 / 2}} \\
& +\frac{\cos \theta}{\left|1+\rho^{2}+h^{2}+2 \rho \sin \theta\right|^{3 / 2}}=0 .
\end{aligned}
$$

Then

$$
\begin{aligned}
& \frac{1}{\sin \theta}\left(\frac{1}{\left|1+\rho^{2}+h^{2}-2 \rho \sin \theta\right|^{3 / 2}}\right. \\
& \left.\quad-\frac{1}{\left|1+\rho^{2}+h^{2}+2 \rho \sin \theta\right|^{3 / 2}}\right) \\
& =\frac{1}{\cos \theta}\left(\frac{1}{\left|1+\rho^{2}+h^{2}-2 \rho \cos \theta\right|^{3 / 2}}\right. \\
& \left.-\frac{1}{\left|1+\rho^{2}+h^{2}+2 \rho \cos \theta\right|^{3 / 2}}\right) .
\end{aligned}
$$


Let

$$
\begin{array}{r}
f(x)=\frac{1}{x}\left(\frac{1}{\left|1+\rho^{2}+h^{2}-2 \rho x\right|^{3 / 2}}\right. \\
\left.-\frac{1}{\left|1+\rho^{2}+h^{2}+2 \rho x\right|^{3 / 2}}\right) ;
\end{array}
$$

then the system (22) is equivalent to $f(\sin \theta)=f(\cos \theta)$. It is obvious that $f(x)$ is even and $f(x)>0$ when $x>0$. We will prove that $f(x)$ is a strictly increasing function when $x>0$.

When $0<x \leq 1$ we compute

$$
\begin{aligned}
\frac{d f(x)}{d x}= & -\frac{1}{x^{2}}\left(\frac{1}{\left|1+\rho^{2}+h^{2}-2 \rho x\right|^{3 / 2}}\right. \\
& +\frac{1}{x}\left(\frac{1}{\left|1+\rho^{2}+h^{2}+2 \rho x\right|^{3 / 2}}\right) \\
= & \frac{3 \rho}{x^{2}\left|1+\rho^{2}+h^{2}-2 \rho x\right|^{5 / 2}} \\
& \left.-\frac{\left(1+h^{2}+\left.2 \rho x\right|^{5 / 2}\left|1+\rho^{2}+h^{2}-2 \rho x\right|^{5 / 2}\right.}{\left|1+\rho^{2}+h^{2}+2 \rho x\right|^{5 / 2}}\right) \\
& +\frac{\left(1+\rho^{2}+h^{2}-2 \rho x\right)\left(1+\rho^{2}+h^{2}+2 \rho x\right)^{5 / 2}}{x^{2}\left|1+\rho^{2}+h^{2}+2 \rho x\right|^{5 / 2}\left|1+\rho^{2}+h^{2}-2 \rho x\right|^{5 / 2}} \\
& +\frac{3 \rho x\left(1+\rho^{2}+h^{2}+2 \rho x\right)^{5 / 2}}{x^{2}\left|1+\rho^{2}+h^{2}+2 \rho x\right|^{5 / 2}\left|1+\rho^{2}+h^{2}-2 \rho x\right|^{5 / 2}}
\end{aligned}
$$

Let

$$
\begin{aligned}
g(x)= & \left(1+\rho^{2}+h^{2}+2 \rho x\right) \\
& \times\left(1+\rho^{2}+h^{2}-2 \rho x\right)^{5 / 2} \\
& -\left(1+\rho^{2}+h^{2}-2 \rho x\right) \\
& \times\left(1+\rho^{2}+h^{2}+2 \rho x\right)^{5 / 2} \\
& +3 \rho x\left(1+\rho^{2}+h^{2}+2 \rho x\right)^{5 / 2} \\
& +3 \rho x\left(1+\rho^{2}+h^{2}-2 \rho x\right)^{5 / 2} .
\end{aligned}
$$

Then

$$
\frac{d f(x)}{d x}=\frac{g(x)}{x^{2}\left|1+\rho^{2}+h^{2}+2 \rho x\right|^{5 / 2}\left|1+\rho^{2}+h^{2}-2 \rho x\right|^{5 / 2}},
$$

$$
g(0)=\left(1+\rho^{2}+h^{2}\right)^{7 / 2}-\left(1+\rho^{2}+h^{2}\right)^{7 / 2}=0,
$$$$
\frac{d g(x)}{d x}=2 \rho\left(1+\rho^{2}+h^{2}-2 \rho x\right)^{5 / 2}
$$$$
-5 \rho\left(1+\rho^{2}+h^{2}+2 \rho x\right)
$$$$
\times\left(1+\rho^{2}+h^{2}-2 \rho x\right)^{3 / 2}
$$$$
+2 \rho\left(1+\rho^{2}+h^{2}+2 \rho x\right)^{5 / 2}
$$$$
-5 \rho\left(1+\rho^{2}+h^{2}-2 \rho x\right)
$$$$
\times\left(1+\rho^{2}+h^{2}+2 \rho x\right)^{3 / 2}
$$$$
+3 \rho\left(1+\rho^{2}+h^{2}+2 \rho x\right)^{5 / 2}
$$$$
+15 \rho^{2} x\left(1+\rho^{2}+h^{2}+2 \rho x\right)^{3 / 2}
$$$$
+3 \rho\left(1+\rho^{2}+h^{2}-2 \rho x\right)^{5 / 2}
$$$$
-15 \rho^{2} x\left(1+\rho^{2}+h^{2}-2 \rho x\right)^{3 / 2},
$$$$
\left.\frac{d g(x)}{d x}\right|_{x=0}=10 \rho\left(1+\rho^{2}+h^{2}\right)^{5 / 2}
$$$$
-10 \rho\left(1+\rho^{2}+h^{2}\right)^{5 / 2}=0,
$$

$$
\begin{aligned}
\frac{d^{2} g(x)}{d x^{2}}= & -10 \rho^{2}\left(1+\rho^{2}+h^{2}-2 \rho x\right)^{3 / 2} \\
& -10 \rho^{2}\left(1+\rho^{2}+h^{2}-2 \rho x\right)^{3 / 2} \\
& +15 \rho^{2}\left(1+\rho^{2}+h^{2}+2 \rho x\right) \\
& \times\left(1+\rho^{2}+h^{2}-2 \rho x\right)^{1 / 2} \\
& +10 \rho^{2}\left(1+\rho^{2}+h^{2}+2 \rho x\right)^{3 / 2} \\
& +10 \rho^{2}\left(1+\rho^{2}+h^{2}+2 \rho x\right)^{3 / 2} \\
& -15 \rho^{2}\left(1+\rho^{2}+h^{2}-2 \rho x\right) \\
& \times\left(1+\rho^{2}+h^{2}+2 \rho x\right)^{1 / 2} \\
& +15 \rho^{2}\left(1+\rho^{2}+h^{2}+2 \rho x\right)^{3 / 2} \\
& +15 \rho^{2}\left(1+\rho^{2}+h^{2}+2 \rho x\right)^{3 / 2} \\
& +45 \rho^{3} x\left(1+\rho^{2}+h^{2}+2 \rho x\right)^{1 / 2}
\end{aligned}
$$




$$
\begin{aligned}
& -15 \rho^{2}\left(1+\rho^{2}+h^{2}-2 \rho x\right)^{3 / 2} \\
& -15 \rho^{2}\left(1+\rho^{2}+h^{2}-2 \rho x\right)^{3 / 2} \\
& +45 \rho^{3} x\left(1+\rho^{2}+h^{2}-2 \rho x\right)^{1 / 2} \\
\geq & 45 \rho^{3} x\left(1+\rho^{2}+h^{2}+2 \rho x\right)^{1 / 2} \\
& +45 \rho^{3} x\left(1+\rho^{2}+h^{2}-2 \rho x\right)^{1 / 2} \\
> & 0, \quad \forall 0<x \leq 1 .
\end{aligned}
$$

Hence by (26), (27), (29), and (30) we have

$$
g(x)>0, \quad \forall 0<x \leq 1
$$

Hence $d f(x) / d x>0$, when $0<x \leq 1$. From $f(\sin \theta)=$ $f(\cos \theta)$ we obtain $|\sin \theta|=|\cos \theta|$; that is, $\theta=(\pi / 4)+$ $(\pi / 2) k$, so $m^{*}$ is on a vertical plane of symmetry.

It is obvious that if the point $m^{*}$ is on a vertical plane of symmetry, $F_{*}$ 's projection on $P$ is directed toward the center of the square.

The proof of Lemma 2 is completed.

2.3. The Proof of Theorem 7. Without loss of generality, suppose that $\widetilde{m}_{1}=\widetilde{m}_{2}=\widetilde{m}_{3}=\widetilde{m}_{4}=m, m_{1}=m_{2}=m_{3}=$ $m_{4}=1$. We can let

$$
\begin{gathered}
q_{1}=(1,0,0), \quad q_{2}=(0,1,0), \\
q_{3}=(-1,0,0), \quad q_{4}=(0,-1,0), \\
\widetilde{q}_{1}=(\rho \cos \varphi, \rho \sin \varphi, h), \\
\widetilde{q}_{2}=\left(\rho \cos \left(\frac{2 \pi}{4}+\varphi\right), \rho \sin \left(\frac{2 \pi}{4}+\varphi\right), h\right), \\
\tilde{q}_{3}=\left(\rho \cos \left(\frac{2 \pi}{4} \times 2+\varphi\right), \rho \sin \left(\frac{2 \pi}{4} \times 2+\varphi\right), h\right), \\
\widetilde{q}_{4}=\left(\rho \cos \left(\frac{2 \pi}{4} \times 3+\varphi\right), \rho \sin \left(\frac{2 \pi}{4} \times 3+\varphi\right), h\right),
\end{gathered}
$$

where $\rho h>0$. It is obvious that $z_{0}=\sum_{j=1}^{4}\left(m_{j} q_{j}+\widetilde{m}_{j}+\right.$ $\left.\tilde{q}_{j}\right) / M=(0,0,4 m h /(4+4 m))=(0,0, m h /(1+m))$. By Lemma 1 , since $\widetilde{m}_{1}, \widetilde{m}_{2}, \widetilde{m}_{3}$, and $\widetilde{m}_{4}$ locate on vertices of a regular polygon, so they form a central configuration; then there exists a constant $\tilde{\lambda}$ (notice that it can be different from $\lambda$ in(16)), such that

$$
\sum_{j \neq k}^{4} \widetilde{m}_{j} \frac{\widetilde{q}_{j}-\widetilde{q}_{k}}{\left|\widetilde{q}_{j}-\widetilde{q}_{k}\right|}=-\tilde{\lambda}\left(\widetilde{q}_{k}-\widetilde{z}_{0}\right),
$$

where $\widetilde{z}_{0}=\left(\sum_{j=1}^{4} \widetilde{q}_{j} \widetilde{m}_{j}\right) / 4 m=(0,0, h)$.
By (1) and (33) we have

$$
\begin{aligned}
-\lambda m \widetilde{P}_{k} & =\sum_{j=1}^{4} m \frac{q_{j}-\widetilde{q}_{k}}{\left|q_{j}-\widetilde{q}_{k}\right|^{3}}+\sum_{j \neq k}^{4} m m \frac{\widetilde{q}_{j}-\widetilde{q}_{k}}{\left|\widetilde{q}_{j}-\widetilde{q}_{k}\right|^{3}} \\
& =\sum_{j=1}^{4} m \frac{P_{j}-\widetilde{P}_{k}}{\left|P_{j}-\widetilde{P}_{k}\right|^{3}}+\sum_{j \neq k}^{4} m m \frac{\widetilde{P}_{j}-\widetilde{P}_{k}}{\left|\widetilde{P}_{j}-\widetilde{P}_{k}\right|^{3}} \\
& =\sum_{j=1}^{4} m \frac{q_{j}-\widetilde{q}_{k}}{\left|q_{j}-\widetilde{q}_{k}\right|^{3}}-\tilde{\lambda}_{m} m\left(\widetilde{q}_{k}-\widetilde{z}_{0}\right) \quad(k=1,2,3,4) .
\end{aligned}
$$

Then

$$
\begin{aligned}
\sum_{j=1}^{4} \frac{m\left(q_{j}-\widetilde{q}_{k}\right)}{\left|q_{j}-\widetilde{q}_{k}\right|^{3}} & \\
\quad= & -\lambda m\left(\widetilde{q}_{k}-z_{0}\right)+\widetilde{\lambda} m\left(\widetilde{q}_{k}-\widetilde{z}_{0}\right) \\
& =m(\widetilde{\lambda}-\lambda) \widetilde{q}_{k}+m\left(0,0, \frac{\lambda m h}{1+m}\right)-m(0,0, \tilde{\lambda} h) .
\end{aligned}
$$

Letting $\widetilde{F_{1}}=\sum_{j=1}^{4} m\left(q_{j}-\widetilde{q}_{1}\right) /\left|q_{j}-\widetilde{q}_{1}\right|^{3}, \widetilde{F_{1}}$ is the force generated by the $m_{1}, m_{2}, m_{3}$, and $m_{4}$.

By (19), $\widetilde{F_{1}}$ 's projection on the plane $P_{1}$ is $(\widetilde{\lambda}-$ $\lambda)(\rho \cos \varphi, \rho \sin \varphi, 0)$, where $P_{1}$ is the plane containing $m_{1}$, $m_{2}, m_{3}$, and $m_{4}$. It is obvious that the projection is directed toward the center of the lower layer regular 4-gons. By Lemma 2 , we have $\varphi=\pi / 4+(\pi / 2) k$ or $\varphi=(\pi / 2) k(k=$ $0,1,2,3)$.

The proof of Theorem 7 is completed.

\section{Acknowledgments}

The authors express their gratitude to Professor Zhang Shiqing for his discussions and helpful suggestions. This work is Supported by NSF of China and Youth Fund of Mianyang Normal University.

\section{References}

[1] S. Zhang and C. Zhu, "Central configurations consist of two layer twisted regular polygons," Science in China A, vol. 45, no. 11, pp. 1428-1438, 2002.

[2] D. G. Saari, Collisions, Rings and Other Newtonian N-body Problems, vol. 104, AMS, Providence, RI, USA, 2005.

[3] A. Wintner, The Analytical Foundations of Celestial Mechanics, Princeton University Press, Princeton, NJ, USA, 1941.

[4] R. Moeckel and C. Simó, "Bifurcation of spatial central configurations from planar ones," SIAM Journal on Mathematical Analysis, vol. 26, no. 4, pp. 978-998, 1995.

[5] Z. F. Xie, S. Q. Zhang, and Q. Zhou, "Two layer regular polygonal centralconfigurations in $\mathrm{R}^{3}$," Preprint, 2000.

[6] D. Bang and B. Elmabsout, "Representations of complex functions, means on the regular $n$-gon and applications to gravitational potential," Journal of Physics A, vol. 36, no. 45, pp. 11435-11450, 2003. 
[7] L. M. Perko and E. L. Walter, "Regular polygon solutions of the $N$-body problem," Proceedings of the American Mathematical Society, vol. 94, no. 2, pp. 301-309, 1985. 


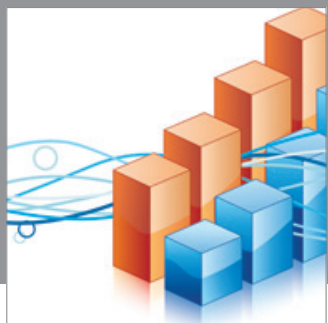

Advances in

Operations Research

mansans

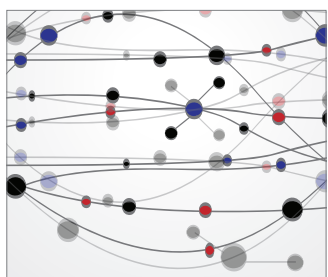

The Scientific World Journal
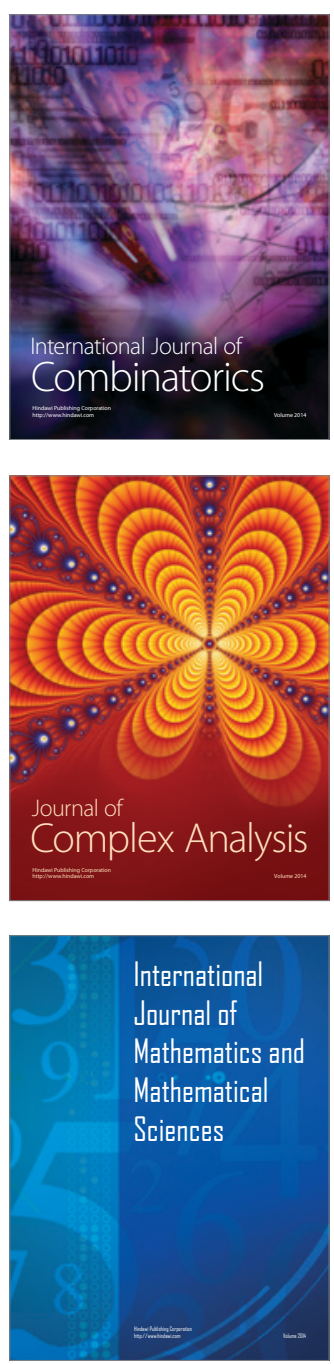
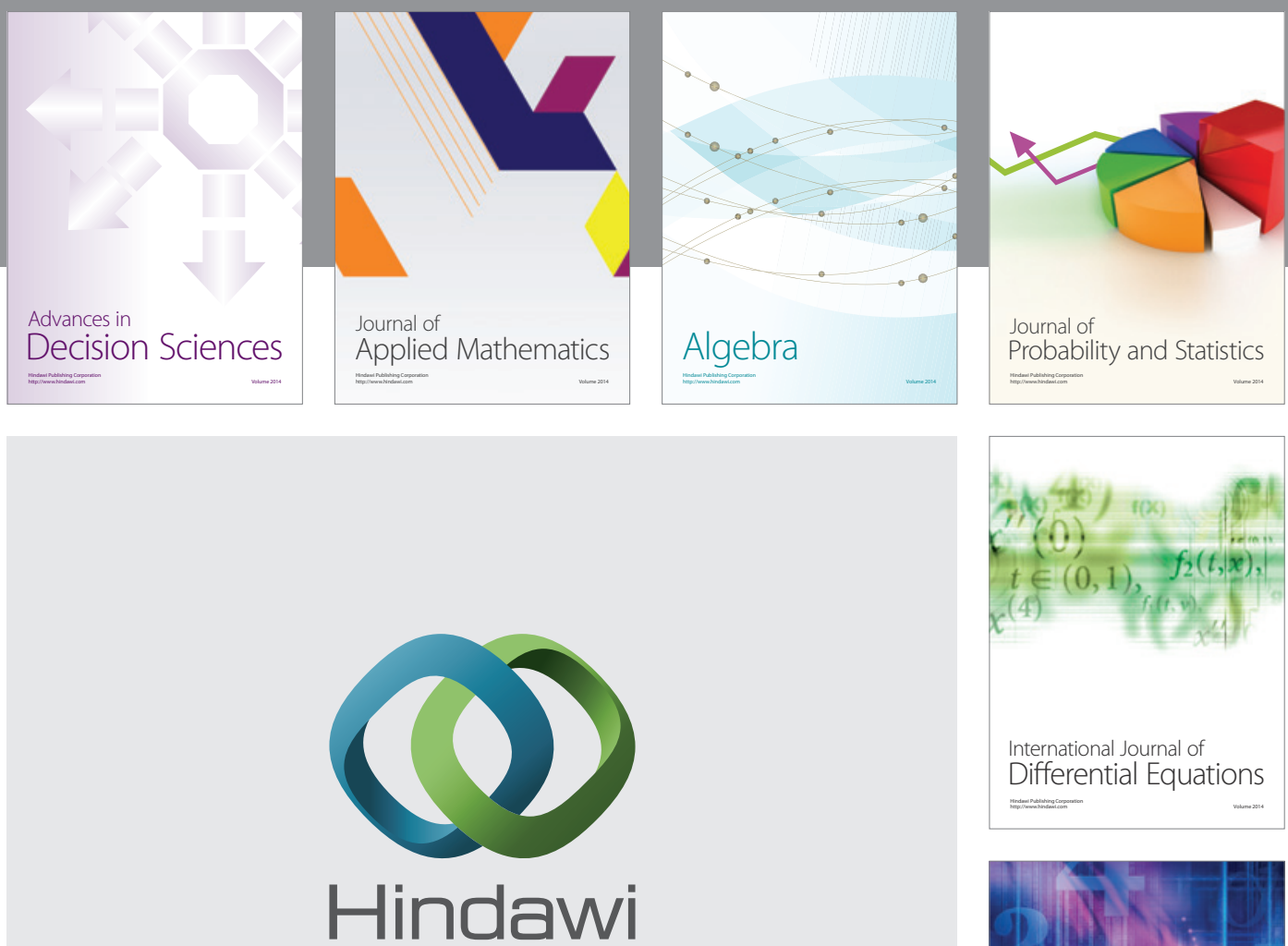

Submit your manuscripts at http://www.hindawi.com
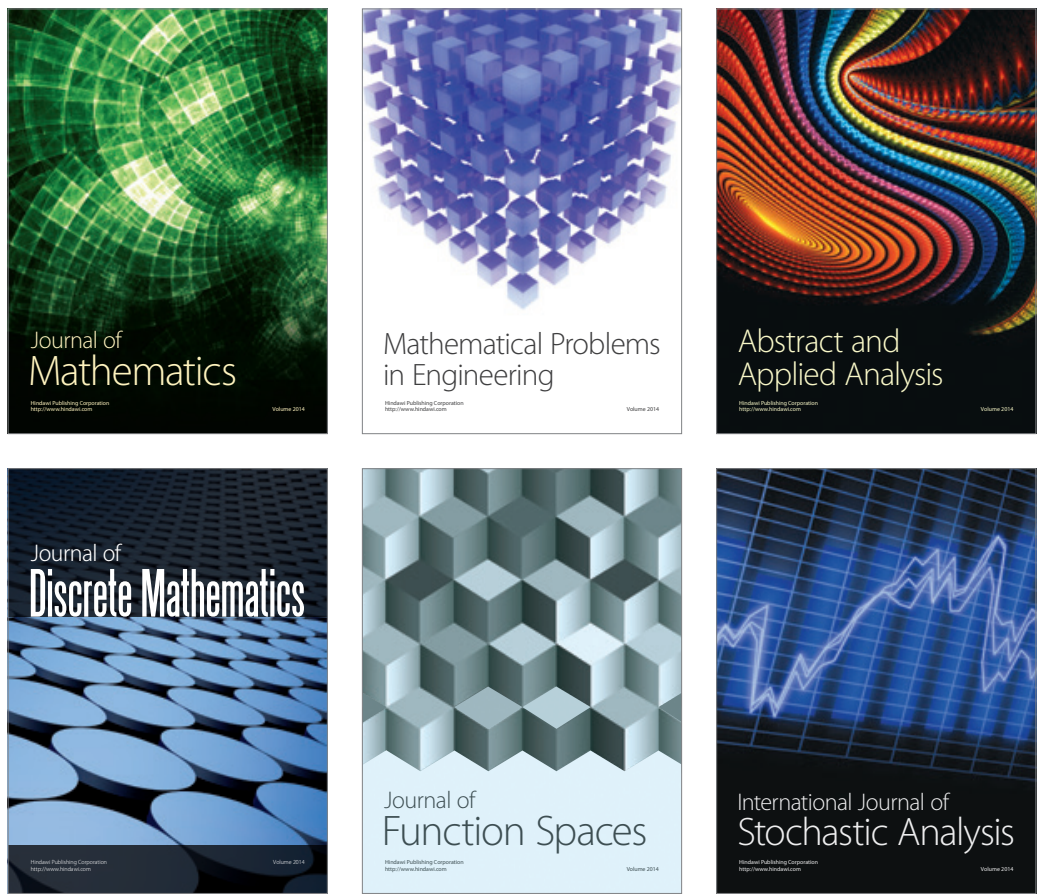

Journal of

Function Spaces

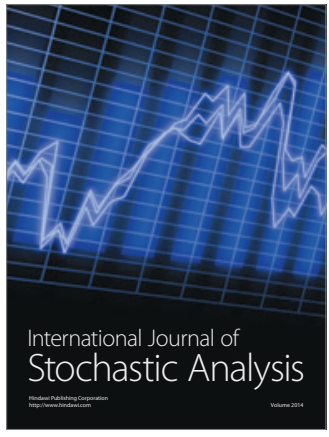

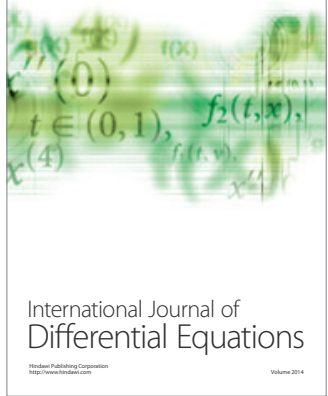
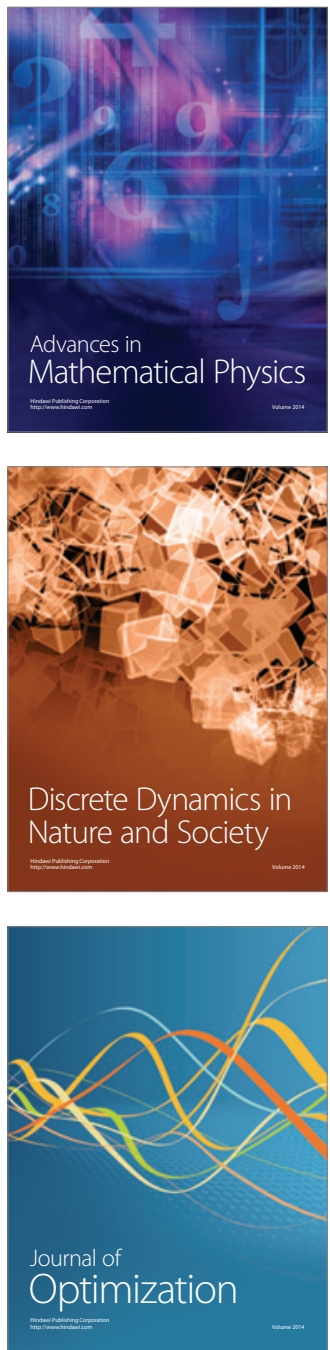